\title{
Impact of Mobility on Convergence Rate in a Wireless Sensor Network
}

\author{
Roya Norouzi Kandalan, Ramanpreet Singh, Kamesh Namuduri, Murali Varanasi, and Bill Buckles \\ Department of Electrical Engineering and \\ Department of Computer Science \\ University of North Texas \\ Denton, Texas \\ royanorouzi@my.unt.edu, ramanpreetsingh@my.unt.edu,kamesh.namuduri@unt.edu,murali.varanasi@unt.edu, \\ bill.buckles@unt.edu
}

\begin{abstract}
Consensus building in wireless sensor networks (WSN's) has been studied extensively over a number of years. However, the role of mobile nodes in a network has not been fully explored. This paper shows that introducing mobility increases the rate of convergence in partially connected and disconnected WSN. It also shows that selection of mobile nodes influences the rate of convergence. The mobile node selection scheme presented here takes advantage of the fact that some nodes are more "central" and "critical" than others and selecting them as mobile nodes can help reach consensus faster. By representing WSN as an undirected graph and analyzing various properties of the nodes, including centrality and position, a simple and effective strategy to select mobile nodes has been presented. Simulation results verify that introducing mobility coupled with an appropriate selection of mobile nodes leads to faster convergence.
\end{abstract}

Keywords-Consensus building; mobile agents; mobility

\section{INTRODUCTION}

Nature is abundant with examples of collective decision making and has inspired many studies. For example, Cronin et al. studied the decision making in ants' and their way of utilizing "wisdom-of-the-crowd" [1]. DeGroot studied the problem of reaching consensus using weighted opinions of individuals having their own subjective probability distribution for the unknown value of some decision making parameter [2]. While DeGroot's work focused on the weighted opinion of all agents, Epstein et al. took into account the structure of the network and showed that hierarchy improves the rate of convergence in a network [3]. Olfati-Saber et al. studied the consensus building in different topologies of directed and undirected networks with planar graph representation and established a direct connection between the algebraic connectivity (eigenvalue) and the performance of linear consensus protocol [4]. Wan et al. established the conditions for convergence in a network represented by a two layered hierarchical graph [5].

In this work, the convergence rate in bipartite networks, in which one group of nodes is mobile and the other is static, is studied. A modified version of system matrix presented in [5] is used in this analysis. A strategy to choose a set of nodes, which can act as effective mobile agents and consequently increase the rate of convergence, is presented.

\section{BIPARTITE NETWORKS}

In a bipartite graph, one end of each edge lies in the set of nodes, while the other end lies in the second set. To represent a WSN as a bipartite graph, the nodes have to be separated into two disjoint sets. In this paper, graph coloring strategy is used to separate the nodes into two different colored groups, such that adjacent nodes do not have the same color. This technique results in a bipartite graph only when no odd cycles are present in the graph. For graphs having odd cycles, a simple algorithm to remove some edges from odd cycles in the graph is used to convert them to bipartite.

In general, the process of removing edges from a graph to eliminate odd cycles is an NP-hard problem, which can be optimized using Goemans-Williamson Algorithm [6]. An alternative to removing edges would be to insert a node in each odd cycle to make the graph two colorable, thus, making it possible to convert into a bipartite graph.

Algorithm
BEGIN Graph-Coloring
1. Initialize two vectors red and black.
2. INSERT the first node in red and SET vector flag to
R.
3. IF vector flag is R, SELECT the next node in red
vector and mark it as processing node
ELSEIF vector flag is B, SELECT the next node in
black vector and mark it as processing node
ENDIF
REPEAT
READ first neighbor of processing node
IF neighbor of processing node does not exist
in black or red
IF vector flag is R, INSERT neighbor in B
ELSEIF vector flag is B, INSERT
neighbor in R
ENDIF
ENDIF


UNTIL all immediate neighbors of processing node are inserted in $\mathrm{R}$ or $\mathrm{B}$

5. IF vector flag is $R$, SET vector flag to $B$ ELSEIF vector flag is B, SET vector flag to $\mathrm{R}$ END IF

6. IF sum of all nodes in $\mathrm{R}$ and $\mathrm{B}$ is equal to the total number of nodes in the graph, STOP ELSE, Go to Step 3

END Graph-coloring

\section{Proposed LEADER-FOLLOWER MODEL}

This paper makes use of the concept of Virtual Fusion Centers (VFCs) as presented in [5]. VFC's are few special nodes in a graph which perform decision making calculations on information gathered from their immediate neighbors. The decision is then transmitted back from the VFC to the sensor nodes. In this paper, we use a leader-follower model which is similar to the VFC model. The important difference is that the leader, unlike a VFC, is a sensor node and contributes to the process of decision making. Guidelines for the selection of leader nodes are discussed in this section.

\section{A. Conditions for convergence}

Consider a network with $n$ sensor nodes where $x[k]=$ $R^{n \times 1}$ denotes the sensor values at iteration $k$. The sensor values at cycle $k+1$ is given by the following Linear Time Invariant (LTI) difference equation:

$$
x[k+1]=A x[k]
$$

where $A$ is referred to as the system matrix and can be calculated using the following relation:

$$
a_{i j}=\frac{1}{L_{i}} \sum_{c} \frac{1}{\operatorname{Deg}\left(L_{c}\right)+1}
$$

$L_{i}$ is the number of mobile nodes in contact with the $i^{t h}$ sensor and $\operatorname{Deg}\left(L_{c}\right)$ is degree of $c^{\text {th }}$ mobile node in contact with $i^{t h}$ and $j^{\text {th }}$ node.

The system matrix shown above is a stochastic matrix, similar to the one presented in [5] and we shall refer to it as the "new" system matrix for the sake of differentiation. The new system matrix can capture the state of a disconnected network which was not the case in [5] and it is calculated by analyzing the graph directly instead of using routing matrix $H$. If $x[0]$ represents the initial sensor values, the sensor data $x[k]$ at iteration $k$ can be represented as follows:

$$
x[k]=A^{k} x[0]
$$

The network is said to have converged when the system matrix doesn't change from the $(k-1)^{t h}$ iteration to the $k^{t h}$ iteration, i.e.

$$
A^{k}=A^{k-1}
$$

To reach consensus, in finite number of iterations, $\lambda_{2}$ must be in the range $[0,1)$ and if the value of $\lambda_{2}$ is small it takes fewer iterations to converge. Notice that this characteristic of the system matrix which is different from that of the Laplacian matrix.

\section{B. Selecting leaders/mobile nodes}

Degree of a node is an important feature to determine its effectiveness as a leader. Higher degree of a node makes the process of gathering and disseminating information faster. In a bipartite graph, the set of nodes having higher degrees will perform better as leaders.

According to Freeman, "a point in a communication network is central to the extent that it falls on the shortest path between pairs of other point" [7]. If all these shortest paths are counted for a given node, it is called node betweenness or betweenness centrality of the node. It is imperative to choose the set of nodes having higher betweenness centrality as the leaders.

Another important feature which influences the selection of leaders is the presence of "leaf" nodes. In a simple unordered tree structure, a node with no children or with only one neighbor is known as leaf node. Leaf nodes do not serve as good leaders; therefore the set of nodes with lesser number of leaf nodes must be chosen as the leader set.

In case selecting a leaf node as leader is unavoidable, it is better to choose a leaf node with shorter path length. The graph shown in Figure 1, has two leaf nodes $S 5$ and $S 8$ and the network converges faster with $S 5$ as a leader than with $S 8$.

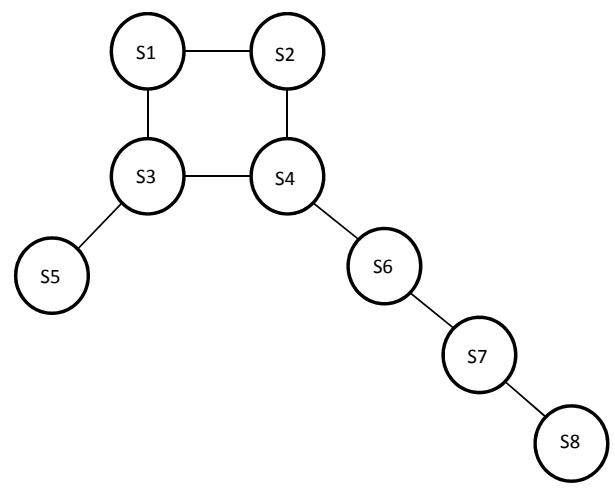

Figure 1: Illustration of leaf nodes

The leader-follower model also works with disconnected graphs, when leader nodes are mobilized (refer to section III.C). 
The second largest eigenvalue $\lambda_{2}$ of system matrix $A$ provides information about algebraic connectivity of the graph [9].

Consider a graph consisting of five sensor nodes $\{S 1, S 2, S 3, S 4, S 5\}$ as shown in Figure 2.

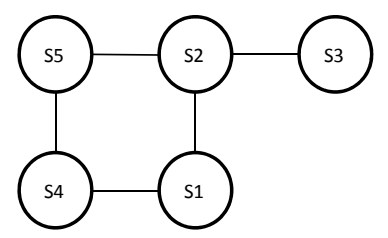

Figure 2: An example with five sensor nodes

Using the algorithm provided in section II, the given set of nodes is divided into two sets: $\{S 2, S 4\}$ and $\{\mathrm{S} 1, \mathrm{~S} 3, \mathrm{~S} 5\}$.

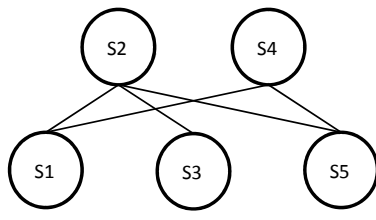

(a)

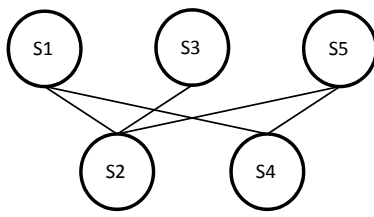

(b)
Figure 3: Graphs (a) and (b) show two possible ways of selecting leaders.

Table 1 shows the average degree of nodes, average node betweenness and number of leaf nodes for both sets of nodes.

Table 1: Criteria for selecting leader nodes

\begin{tabular}{|c|c|c|c|}
\hline $\begin{array}{c}\text { Set of } \\
\text { Nodes }\end{array}$ & $\begin{array}{c}\text { Average } \\
\text { Degree } \\
\text { of Nodes }\end{array}$ & $\begin{array}{c}\text { Average } \\
\text { Node } \\
\text { Betweenness }\end{array}$ & $\begin{array}{c}\text { Number } \\
\text { of Leaf } \\
\text { Nodes }\end{array}$ \\
\hline$S 2, S 4$ & 2.5 & 0.3 & 0 \\
\hline $\begin{array}{c}S 1, S 3, \\
S 5\end{array}$ & 1.66 & 0.066 & 1 \\
\hline
\end{tabular}

According to the selection criteria, it is clear that $S 2$ and $S 4$ will serve as better leaders. The validity of this selection criteria is illustrated in Figure 4 which shows the improvement in algebraic connectivity and therefore in the rate of convergence.

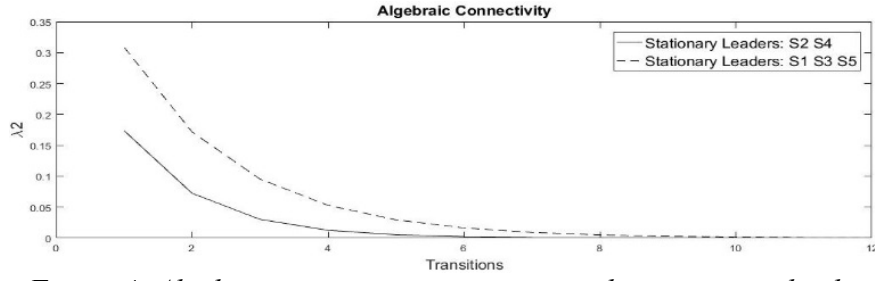

Figure 4: Algebraic connectivity improves with appropriate leader selection

\section{Super graph as a collection of instantaneous graphs}

At any iteration $k$, the state of the network can be represented by system matrix $A_{k}$. As shown in [8], the individual graphs may be disconnected, but the supergraph representing the collection of these individual graphs may become connected after several iterations. The system matrix of the supergraph is given by the product of the system matrices corresponding to the instantaneous graphs.

Consider an example of three sensor nodes which form a set of two realizable graphs given by $G=\left\{G_{1}, G_{2}\right\}$ and have corresponding system matrices $A_{1}$ and $A_{2}$. The supergraph of all the realizable graphs is given by $\Gamma\left(\left\{G_{1}, G_{2}\right\}\right)$. As it can be seen in Figure 5, each individual graph $G_{1}$ and $G_{2}$ is disconnected while the supergraph $\Gamma(G)$ is connected. The system matrix of the supergraph $A$ is given by:

$$
A=A_{1} A_{2}
$$

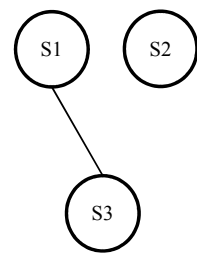

(a)

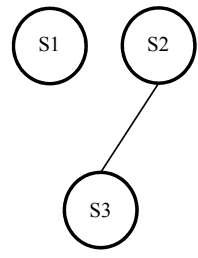

(b)

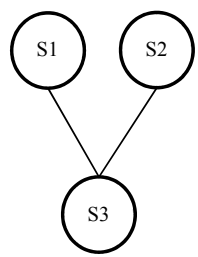

(c)
Figure 5: (a) and (b) are disconnected graphs given by $G_{1}$ and $\mathrm{G}_{2}$; (c) represents the supergraph

\section{DETERMINISTIC MOBILITY}

Once a network is converted into a bipartite graph, one of the two groups can be made mobile. Mobile nodes can move in either deterministic or random manner. To show the effect of a good selection of mobile nodes, a deterministic round robin mobility model is considered. Consider a network with seven sensor nodes $\{\mathrm{S} 1, \mathrm{~S} 2, \mathrm{~S} 3, \mathrm{~S} 4, \mathrm{~S} 5, \mathrm{~S} 6, \mathrm{~S} 7\}$. Using the graph coloring algorithm, the network is divided into two groups of nodes: $\{\mathrm{S} 1, \mathrm{~S} 5\}$ and $\{\mathrm{S} 2, \mathrm{~S} 3, \mathrm{~S} 4, \mathrm{~S} 6, \mathrm{~S} 7\}$, which leads to two possible ways to select mobile nodes. In case of round robin mobility, if the number of mobile nodes is $m$, the state of the network can be captured by system matrices $A_{1}, A_{2} A_{3}, \ldots A_{m}$. A "transition" is the change in state of the network due to movement of the nodes. One or more transitions can occur in one iteration as seen in the two cases below.

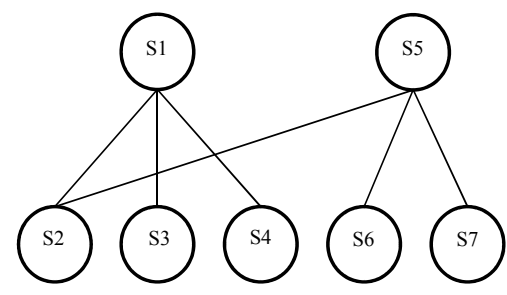

Figure 6: Partially connected network with SI and S5 as leaders 


\section{A. Case I:}

In the first case, $S 1$ and $S 5$ are mobile and the two nodes move in round robin order as shown in Figure 6. For each movement of $S 1$ and $S 5$, a new system matrix is used to represent the network. In this case, since $m=2$, only two system matrices $A_{1}$ and $A_{2}$ are possible. The movement of the nodes changes the structure of the network and the system matrix of the graph at different iterations is given by equation (3) which is modified to (6).

$$
\begin{gathered}
\boldsymbol{A}_{k=0}=A_{0} \\
\boldsymbol{A}_{k=1}=A_{0} A_{1}
\end{gathered}
$$

The error value in each iteration is given by (4) [8],

$$
e_{i}=\left\|A_{0} A_{1} \ldots A_{i}-J\right\|
$$

where $J$ is the final consensus matrix.. Figure 7 shows the improvement of convergence rate when the leaders are moving.

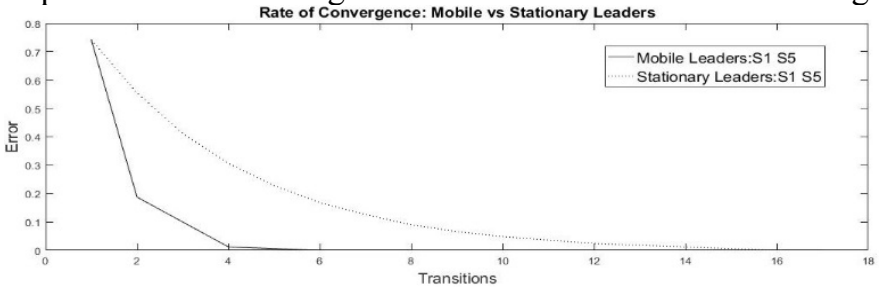

Figure 7: Improvement in rate of convergence due to mobility.

As shown in Figure 8, the algebraic connectivity of the graph improves with the movement of $S 1$ and $S 5$.

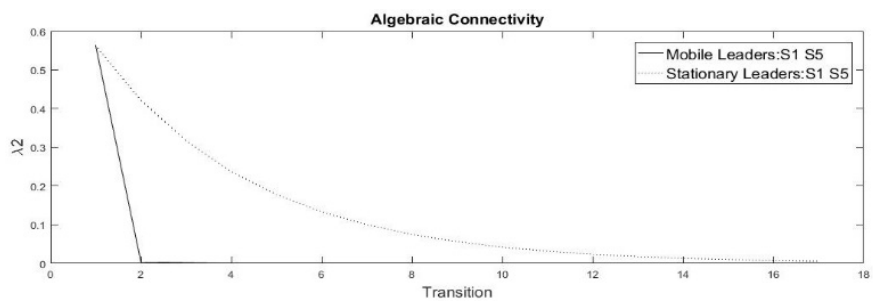

Figure 8: Improvement in algebraic connectivity $\left(\lambda_{2}\right)$ due to mobility.

\section{B. Case 2:}

In this case, similar to the first group, the second group of nodes $\{\mathrm{S} 2, \mathrm{~S} 3, \mathrm{~S} 4, \mathrm{~S} 6, \mathrm{~S} 7\}$, is made mobile which leads to five distinct matrices $A_{1}, A_{2}, A_{3}, A_{4}, A_{5}$ and the resulting error is calculated for each transition.

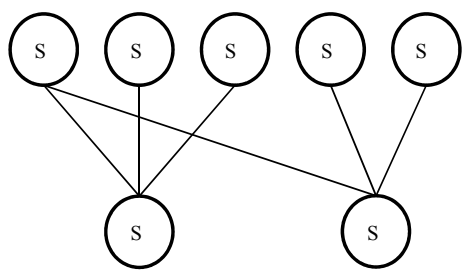

Figure 9: Partially Connected network with S2, S3, S4, S6 and S7 as leaders

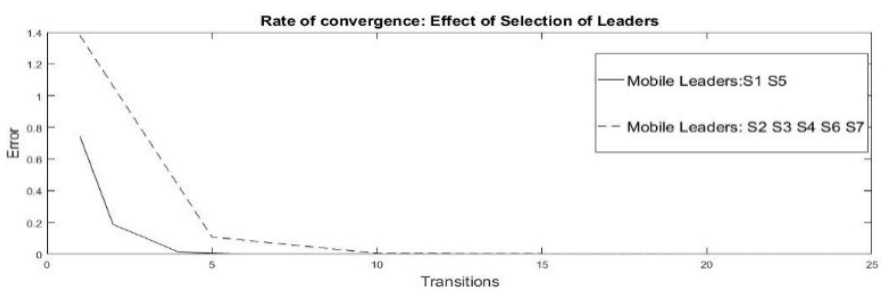

Figure 10: Improvement in rate of convergence with appropriate leader selection

It is clear from Figure 10 that the total number of transitions to reach consensus is 6 , when $\{\mathrm{S} 1, \mathrm{~S} 5\}$ are selected as leaders, while it is 15 when $\{\mathrm{S} 2, \mathrm{~S} 3, \mathrm{~S} 4, \mathrm{~S} 6, \mathrm{~S} 7\}$ are selected as leaders. It clearly shows a $60 \%$ improvement over the number of transitions required to reach consensus in this case.

\section{CONCLUSIONS}

In this work, a leader-follower model for consensus building is presented. A simple algorithm which converts any graph, including graphs having odd cycles, to a bipartite graph has been presented. Through simulations, it is demonstrated that following the criteria presented in this work, one set of nodes can be more effective as leaders than the other set of nodes. Simulation results show that mobility significantly improves the rate of convergence for partially connected and disconnected networks.

\section{REFERENCES}

[1] A. L. Cronin and M. C. Stumpe, "Ants work harder during desicionmaking in small group," Journal of the royal society interface, 2014.

[2] M. H. Degroot, "Reaching a Consensus," Journal of the American statistical assosiation, vol. 69, no. 345, pp. 118-121, 1974.

[3] M. Epstein, K. Lynch, K. Johansson and R. M. Murry, "Using Hierarchial Decomposition to speed up average consensus".

[4] a. R. M. M. Reza Olfati-Saber, "Consensus Problems in Networks of Agents With Switching Topology and Time-Delays," IEEE TRANSACTIONS ON AUTOMATIC CONTROL, vol. 49, pp. 15201533, 2004

[5] Y. Wan, K. Namuduri, S. Akula and M. Varanasi, "The Impact of Multi-group Multi-layer network structure on the performance of disributed consensus building strategies," International Journal of Robost and Nonlinear Control, vol. 23, pp. 653-662, 2013.

[6] M. X. Goemans and D. P. Williamson, "Improved Approximation Algorithm for Maximum Cut and Satisfactory Problem Using Semidefinite Programming," Journal of the Association for ComputingMachinery, vol. 42, pp. 1115-114, 1995.

[7] L. C. Freeman, " A Set of Measures of Centrality," Sociometry, vol. 40, no. 1, pp. 35-41, 1977.

[8] D. Bajovic, J. Xavier, J. M. F. Moura, B. Sinopoli, "Consensus and products of random stochastic matrices: Exact rate for convergence in probability", IEEE Trans. Signal Process., vol. 61, no. 10, pp. 2557-2571, May 2013.

[9] C. Godsil and G. Royle, Algebraic Graph Theory, New York: Springer- Verlag, 2001. 